\title{
THE SHARP INTERFACE LIMIT OF A PHASE FIELD MODEL FOR MOVING CONTACT LINE PROBLEM*
}

\author{
XIAO-PING WANG ${ }^{\dagger}$ AND YA-GUANG WANG W $^{\ddagger}$
}

\begin{abstract}
Using method of matched asymptotic expansions, we derive the sharp interface limit for the diffusive interface model with the generalized Navier boundary condition recently proposed by Qian, Wang and Sheng in $[9,11]$ for the moving contact line problem. We show that the leading order problem satisfies a boundary value problem for a coupled Hale-Shaw and Navier-Stokes equations with the interface being a free boundary, and the leading order dynamic contact angle is the same as the static one satisfying the Young's equation.
\end{abstract}

Key words. Sharp interface limit, matched asymptotic expansion, moving contact line.

AMS subject classifications. 76T05, 34E05

1. Introduction. The modeling of two phase immiscible fluid flow over a solid surface has been a challenging problem. It was shown in [6] that the classical no-slip boundary condition leads to nonphysical contact-line singularity, or infinite viscous dissipation near the moving contact line. The recent discovery of the generalized Navier boundary condition (GNBC) has resolved this issue with respect to immiscible flow over flat surfaces, by showing that the continuum calculations based on the new slip boundary condition can quantitatively reproduce MD simulation results $[9,11]$. The GNBC states that the relative slip velocity between the fluid and the solid wall is proportional to the total tangential stress - the sum of the viscous stress and the uncompensated Young stress, the latter arises from the deviation of the fluid-fluid interface from its static configuration. Combining the GNBC with the Cahn-Hilliard $(\mathrm{CH})$ equation [3], which has been used to formulate a phase-field description of immiscible two-phase flows [13, 5, 7, 2], and the Navier-Stokes equations, a continuum hydrodynamic model was proposed by Qian, Wang and Sheng in [9]. The CahnHilliard free energy is given by

$$
F(\phi)=\int_{\Omega}\left\{\frac{1}{2} K|\nabla \phi|^{2}+f(\phi)\right\} d \mathbf{r}
$$

Here $\phi(\mathbf{r})$ is the phase field, locally defined from $\phi=\left(\rho_{2}-\rho_{1}\right) /\left(\rho_{2}+\rho_{1}\right)$, with $\rho_{1}$ and $\rho_{2}$ being the local densities of the two fluid species; $f(\phi)=\frac{u}{4}\left(\phi^{2}-\frac{r}{u}\right)^{2}$ is a double well potential, in which $K, r$, and $u$ are parameters that can be determined from the interfacial thickness $\xi=\sqrt{K / u}$, the interfacial tension $\gamma=2 \sqrt{2} r^{2} \xi / 3 u$, and the two homogeneous equilibrium phases $\phi_{ \pm}= \pm \sqrt{r / u}$, all measurable in MD simulations. The two coupled equations of motion are the convection-diffusion equation for the composition field $\phi(\mathbf{r})$ and the Navier-Stokes equation in the presence of the capillary force density:

$$
\frac{\partial \phi}{\partial t}+\mathbf{v} \cdot \nabla \phi=M \nabla^{2} \mu
$$

\footnotetext{
*Received December 30, 2007; accepted for publication June 3, 2008.

${ }^{\dagger}$ Department of Mathematics, Hong Kong University of Science and Technology, Clear Water Bay, Kowloon, Hong Kong, China (mawang@ust.hk).

${ }_{\ddagger}$ Department of Mathematics, Shanghai Jiao Tong University, 200240 Shanghai, China (ygwang@ sjtu.edu.cn).
} 


$$
\rho\left[\frac{\partial \mathbf{v}}{\partial t}+(\mathbf{v} \cdot \nabla) \mathbf{v}\right]=-\nabla p+\nabla \cdot \boldsymbol{\sigma}^{v}+\mu \nabla \phi+\mathbf{f}_{e}
$$

together with the incompressibility condition $\nabla \cdot \mathbf{v}=0$. Here $\mu=\delta F / \delta \phi$ is the chemical potential derived from the $\mathrm{CH}$ free energy functional $F, M$ is the mobility coefficient, $\rho$ is the mass density of the fluid, $p$ is the pressure, $\boldsymbol{\sigma}^{v}=\eta\left[(\nabla \mathbf{v})+(\nabla \mathbf{v})^{T}\right]$ is the Newtonian viscous stress tensor with $\eta$ being the viscosity, $\mu \nabla \phi$ is the capillary force density, and $\mathbf{f}_{e}$ is the external force. The boundary conditions at the solid surface are the impermeability conditions $\partial_{n} \mu=0, v_{n}=0$, the relaxational equation for phases $\phi$ :

$$
\frac{\partial \phi}{\partial t}+v_{\tau} \partial_{\tau} \phi=-\Gamma L(\phi),
$$

and the GNBC in continuum differential form:

$$
\beta(\phi) v_{\tau}^{\text {slip }}=-\eta\left(\partial_{n} v_{\tau}+\partial_{\tau} v_{n}\right)+L(\phi) \partial_{\tau} \phi .
$$

Here $\tau$ denotes the direction tangent to the solid surface (for two-dimensional flows), $n$ denotes the outward surface normal, $\Gamma$ is a positive phenomenological parameter, $L(\phi)=K \partial_{n} \phi+\partial \gamma_{w f}(\phi) / \partial \phi$ with $\gamma_{w f}(\phi)$ being the fluid-solid interfacial free energy per unit area, $\beta(\phi)$ is the slip coefficient which may locally depend on phases $\phi$, and $L(\phi) \partial_{\tau} \phi$ is the uncompensated Young stress. The numerical simulations based on this new model have also shown remarkable agreement with the molecular dynamic simulations $[9,10]$, indicating that the new model gives the correct mechanism for the motion of the contact line. More recently, it is shown in [11] that the GNBC can also be derived from the principle of minimum energy dissipation, formulated by Onsager for small perturbations away from equilibrium.

The main purpose of this note is to study the sharp interface limit for the phase field model presented above. If one chooses $K=\epsilon$ and $r=u=\frac{1}{\epsilon}$, then the interface thickness is $\xi=\epsilon$, and the two homogeneous equilibrium phases are $\phi_{ \pm}= \pm 1$. We assume that the mobility coefficient $M$ and the viscosity $\eta$ are positive constants independent of the interface thickness $\epsilon$ while the relaxation parameter is assumed to be $\Gamma=\frac{1}{\epsilon}$. The cases that $M$ and $\eta$ are $\epsilon$ dependent will be discussed separately at the end of the paper. We consider the motion of two phase immiscible fluids in a channel $\Omega=\{x \in \mathbb{R}, 0 \leq y \leq L\}$. The equations (2), (3) and the incompressibility equation become

$$
\left\{\begin{array}{l}
\partial_{t} \phi^{\epsilon}+\left(v^{\epsilon} \cdot \nabla\right) \phi^{\epsilon}=M \Delta \mu^{\epsilon} \\
\partial_{t} v^{\epsilon}+\left(v^{\epsilon} \cdot \nabla\right) v^{\epsilon}+\nabla p^{\epsilon}=\eta \Delta v^{\epsilon}+\mu^{\epsilon} \nabla \phi^{\epsilon} \\
\nabla \cdot v^{\epsilon}=0
\end{array}\right.
$$

with

$$
\mu^{\epsilon}=f^{\prime}\left(\phi^{\epsilon}\right)-\epsilon \Delta \phi^{\epsilon}
$$

being the chemical potential. The GNBC and the other boundary conditions at the 
solid boundaries $\partial \Omega=\{y=0, L\}$ are

$$
\left\{\begin{array}{l}
\beta v_{1}^{\epsilon}+\eta \frac{\partial v_{1}^{\epsilon}}{\partial n}=\frac{\partial \phi^{\epsilon}}{\partial x}\left(\epsilon \frac{\partial \phi^{\epsilon}}{\partial n}+\frac{\partial \gamma_{w f}\left(\phi^{\epsilon}\right)}{\partial \phi}\right) \\
v_{2}^{\epsilon}=0 \\
\frac{\partial \mu^{\epsilon}}{\partial y}=0 \\
\frac{\partial \phi^{\epsilon}}{\partial t}+v_{1}^{\epsilon} \frac{\partial \phi^{\epsilon}}{\partial x}=-\frac{1}{\epsilon}\left(\epsilon \frac{\partial \phi^{\epsilon}}{\partial n}+\frac{\partial \gamma_{w f}\left(\phi^{\epsilon}\right)}{\partial \phi}\right)
\end{array}\right.
$$

where $\vec{n}$ is the unit outward normal vector on $\partial \Omega, \beta$ is the slipping coefficient and is assumed to be a constant for simplicity.

In the absence of fluid flow and assuming the interface does not intersect with the physical boundary $\partial \Omega$, the problem (6) is simplified to the classical Cahn-Hilliard equation for the phase-field $\phi^{\epsilon}$ with the boundary conditions $\frac{\partial \phi^{\epsilon}}{\partial n}=0$ and $\frac{\partial \mu^{\epsilon}}{\partial n}=0$ at $\partial \Omega$. The sharp interface limit of this problem is studied by Pego [8] using the method of matched asymptotic expansion. As the interface thickness vanishes, the limit behavior of the chemical potential can be described by a boundary value problem for the Hele-Shaw equation with the interface being a free boundary, and the normal velocity of the interface is proportional to the jump of the normal derivatives of the chemical potential on the interface. In [1], Alikakos, Bates and Chen rigorously justified Pego's asymptotic analysis under certain conditions.

In this paper, we are interested in the sharp interface limit of two phase fluid system (6) with moving contact line (when the interface intersects with physical boundary). Using method of matched asymptotic expansions, we derived the equations that describe the solution away from the interface $\Gamma^{0}$. We also derive the dynamical equation of the interface and the transmission conditions of outer flows at the interface. We conclude that the leading profiles satisfy a boundary value problem for the coupled Hale-Shaw and Navier-Stokes equations with the interface being a free boundary, and the leading order contact angle equals to the static one satisfying the Young's equation.

2. The matched asymptotic expansion in the sharp interface limit. We study the asymptotic behavior of solutions to the problem (6)-(8) in the limit as the interface thickness $\epsilon \rightarrow 0$. We denote the domain by $\Omega_{T}=\{t \in[0, T], x \in \mathbb{R}, 0 \leq$ $y \leq L\}$, and the interface by $\Gamma^{\epsilon}=\left\{(t, x, y) \in \Omega_{T} \mid \phi^{\epsilon}(t, x, y)=0\right\}$. Let $d^{\epsilon}(t, x, y)$ be the spatial signed distance from $(t, x, y) \in \Omega_{T}$ to $\Gamma^{\epsilon},\left|\nabla d^{\epsilon}\right|=1$ with $\nabla$ denoting the gradient operator in the spatial variables. Suppose that $d^{\epsilon}(t, x, y)$ has the expansion

$$
d^{\epsilon}(t, x, y)=\sum_{j \geq 0} \epsilon^{j} d^{j}(t, x, y)
$$

then we have $\left|\nabla d^{0}\right|=1$, so $d^{0}(t, x, y)$ is the spatial signed distance from the point $(t, x, y) \in \Omega_{T}^{ \pm}=\left\{ \pm d^{0}(t, x, y)>0\right\}$ to $\Gamma^{0}=\left\{(t, x, y) \in \Omega_{T} \mid d^{0}(t, x, y)=0\right\}$, the sharp limit of the interface $\Gamma^{\epsilon}$.

2.1. The outer expansion. First, we consider the outer expansion away from the interface in the domains $\Omega_{T}^{ \pm} \backslash \Gamma^{0}$ :

$$
\left(\phi^{\epsilon}, \mu^{\epsilon}, v^{\epsilon}, p^{\epsilon}\right)=\sum_{j \geq 0} \epsilon^{j}\left(\phi_{j}^{ \pm}, \mu_{j}^{ \pm}, v_{j}^{ \pm}, p_{j}^{ \pm}\right)(t, x, y)
$$


From the equations in (6), one easily obtain that in the leading order, $\phi_{0}^{ \pm}= \pm 1$,

$$
\left\{\begin{array}{l}
\Delta \mu_{0}^{ \pm}=0, \\
\partial_{t} v_{0}^{ \pm}+\left(v_{0}^{ \pm} \cdot \nabla\right) v_{0}^{ \pm}+\nabla p_{0}^{ \pm}=\eta \Delta v_{0}^{ \pm}, \\
\nabla \cdot v_{0}^{ \pm}=0 .
\end{array}\right.
$$

For $j \geq 1, \mu_{j}^{ \pm}$satisfies a Poisson equation, and $\left(v_{j}^{ \pm}, p_{j}^{ \pm}\right)$satisfy linearized incompressible Navier-Stokes equations with a forcing term coming from the capillary force.

The leading order boundary conditions in (8) gives

$$
v_{0,2}^{ \pm}=0, \quad \beta v_{0,1}^{ \pm}+\eta \frac{\partial v_{0,1}^{ \pm}}{\partial n}=0, \quad \frac{\partial \mu_{0}^{ \pm}}{\partial y}=0 \quad \text { on } \quad\{y=0, L\}
$$

To solve the leading order outer solution from (10) and (11), we need to find the equation of the interface $\Gamma^{0}$, and the transmission conditions of $\mu_{0}^{ \pm}$and $\left(v_{0}^{ \pm}, p_{0}^{ \pm}\right)$on $\Gamma^{0}$. These will be derived from the inner expansion near the interface $\Gamma^{0}$.

2.2. The inner expansion. We now study the asymptotic behavior of solutions $\left(\phi^{\epsilon}, \mu^{\epsilon}, v^{\epsilon}, p^{\epsilon}\right)$ to the problem (6)-(8) in a neighborhood of the interface $\Gamma^{0}$, and derive equations for inner layer profiles.

In a small neighborhood of $\Gamma^{0}$, consider inner expansion of the following form:

$$
\left(\phi^{\epsilon}, \mu^{\epsilon}, v^{\epsilon}, p^{\epsilon}\right)=\left(\tilde{\phi}^{\epsilon}, \tilde{\mu}^{\epsilon}, \tilde{v}^{\epsilon}, \tilde{p}^{\epsilon}\right)\left(t, x, y, \frac{d^{\epsilon}(t, x, y)}{\epsilon}\right)
$$

where

$$
\left(\tilde{\phi}^{\epsilon}, \tilde{\mu}^{\epsilon}, \tilde{v}^{\epsilon}, \tilde{p}^{\epsilon}\right)=\sum_{j \geq 0} \epsilon^{j}\left(\phi^{j}, \mu^{j}, v^{j}, p^{j}\right)(t, x, y, \xi)
$$

with $\xi=\frac{d^{\epsilon}}{\epsilon}$, and $\left(\phi^{j}, \mu^{j}, v^{j}, p^{j}\right)$ goes to a limit exponentially when $\xi \rightarrow \pm \infty$.

As in [4], we requiring the following matching conditions for the inner and outer expansions:

$$
\sum_{j \geq 0} \epsilon^{j} \phi^{j}\left(t, x, y, \frac{d^{\epsilon}}{\epsilon}\right) \approx \sum_{j \geq 0} \epsilon^{j} \phi_{j}^{ \pm}(t, x, y) .
$$

Therefore, we have

$$
\left\{\begin{array}{l}
\lim _{\xi \rightarrow \pm \infty} \phi^{0}(t, \bar{x}, \bar{y}, \xi)=\phi_{0}^{ \pm}(t, \bar{x}, \bar{y}) \\
\lim _{\xi \rightarrow \pm \infty}\left(\phi^{1}(t, \bar{x}, \bar{y}, \xi)-\left(\phi_{1}^{ \pm}+\xi \nabla d^{0} \cdot \nabla \phi_{0}^{ \pm}\right)(t, \bar{x}, \bar{y})\right)=0
\end{array}\right.
$$

for all fixed $(t, \bar{x}, \bar{y}) \in \Gamma^{0}$, which implies

$$
\lim _{\xi \rightarrow \pm \infty} \partial_{\xi} \phi^{1}(t, \bar{x}, \bar{y}, \xi)=\left(\nabla d^{0} \cdot \nabla\right) \phi_{0}^{ \pm}(t, \bar{x}, \bar{y}) .
$$

Similar matching conditions hold for $\left(\mu^{0}, \mu^{1}\right),\left(v^{0}, v^{1}\right)$ and $\left(p^{0}, p^{1}\right)$ with some obvious changes of notations. 
Inserting the ansatz (12) into the equations in (6), we easily obtain

$$
\begin{aligned}
& \tilde{\phi}_{\xi \xi}^{\epsilon}+\epsilon\left(\Delta d^{\epsilon} \tilde{\phi}_{\xi}^{\epsilon}+2\left(\nabla d^{\epsilon} \cdot \nabla\right) \tilde{\phi}_{\xi}^{\epsilon}\right)-\left(\left(\tilde{\phi}^{\epsilon}\right)^{3}-\tilde{\phi}^{\epsilon}\right)+\epsilon^{2} \Delta \tilde{\phi}^{\epsilon}+\epsilon \tilde{\mu}^{\epsilon}=0 \\
& M \tilde{\mu}_{\xi \xi}^{\epsilon}+\epsilon\left\{M\left(\Delta d^{\epsilon}+2\left(\nabla d^{\epsilon} \cdot \nabla\right)\right) \tilde{\mu}_{\xi}^{\epsilon}-\left(\partial_{t} d^{\epsilon}+\left(\tilde{v}^{\epsilon} \cdot \nabla\right) d^{\epsilon}\right) \tilde{\phi}_{\xi}^{\epsilon}\right\} \\
& +\epsilon^{2}\left(M \Delta \tilde{\mu}^{\epsilon}-\partial_{t} \tilde{\phi}^{\epsilon}-\left(\tilde{v}^{\epsilon} \cdot \nabla\right) \tilde{\phi}^{\epsilon}\right)=0 \\
& \eta \tilde{v}_{\xi \xi}^{\epsilon}-\epsilon\left\{\left(\partial_{t} d^{\epsilon}+\left(\tilde{v}^{\epsilon} \cdot \nabla\right) d^{\epsilon}\right) \tilde{v}_{\xi}^{\epsilon}-\eta\left(\Delta d^{\epsilon}+2 \nabla d^{\epsilon} \cdot \nabla\right) \tilde{v}_{\xi}^{\epsilon}+\left(\tilde{p}_{\xi}^{\epsilon}-\tilde{\mu}^{\epsilon} \tilde{\phi}_{\xi}^{\epsilon}\right) \nabla d^{\epsilon}\right\} \\
& =\epsilon^{2}\left(\partial_{t} \tilde{v}^{\epsilon}+\left(\tilde{v}^{\epsilon} \cdot \nabla\right) \tilde{v}^{\epsilon}+\nabla \tilde{p}^{\epsilon}-\eta \Delta \tilde{v}^{\epsilon}-\tilde{\mu}^{\epsilon} \nabla \tilde{\phi}^{\epsilon}\right) \\
& \left(\tilde{v}_{\xi}^{\epsilon} \cdot \nabla\right) d^{\epsilon}+\epsilon \nabla \cdot \tilde{v}^{\epsilon}=0
\end{aligned}
$$

From the leading order term of (17), it follows immediately that

$$
\mu^{0}, v^{0} \text { are independent of } \xi \text {. }
$$

From the zero-th order of the equations (17)(18), and the inner-outer matching conditions (14) we deduce that the outer solutions $\mu_{0}^{ \pm}$and $v_{0}^{ \pm}$are continuous across the interface $\Gamma^{0}$. The leading order term in equation (16) gives the inner layer profile

$$
\left\{\begin{array}{l}
\phi_{\xi \xi}^{0}=\left(\phi^{0}\right)^{3}-\phi^{0} \\
\left.\phi^{0}\right|_{\xi=0}=0, \quad \lim _{\xi \rightarrow \pm \infty} \phi^{0}= \pm 1
\end{array}\right.
$$

which uniquely determines $\phi=\phi^{0}(\xi)$ being independent of $(t, x, y)$.

On the other hand, from the next order term of (17) we have

$$
\left(\partial_{t} d^{0}+v^{0} \cdot \nabla d^{0}\right) \phi_{\xi}^{0}=M\left(\mu_{\xi \xi}^{1}+\Delta d^{0} \mu_{\xi}^{0}+2 \nabla d^{0} \cdot \nabla \mu_{\xi}^{0}\right) .
$$

Integrating the equation (22) with respect to $\xi$ over $(-\infty,+\infty)$ and using the matching condition (15) for $\mu^{1}$, we deduce the evolution equation for the interface $\Gamma^{0}=\left\{d^{0}(t, x, y)=0\right\}$ :

$$
\partial_{t} d^{0}+v_{0}^{+} \cdot \nabla d^{0}=\frac{M}{2} \nabla d^{0} \cdot\left[\nabla \mu_{0}\right] .
$$

Furthermore, from (16) we know that $\phi^{1}(t, x, y, \xi)$ satisfies the problem

$$
\left\{\begin{array}{l}
\phi_{\xi \xi}^{1}-\left(3\left(\phi^{0}\right)^{2}-1\right) \phi^{1}=-\mu^{0}-\Delta d^{0} \phi_{\xi}^{0} \\
\phi^{1}(t, x, y, 0)=0, \quad \phi^{1}(t, x, y, \cdot) \in L^{\infty}(\mathbb{R}) .
\end{array}\right.
$$

The solvability condition for $\phi^{1}$ gives

$$
\int_{\mathbb{R}}\left(\mu^{0}+\Delta d^{0} \phi_{\xi}^{0}\right) \phi_{\xi}^{0} d \xi=0
$$

which implies

$$
\mu_{0}^{ \pm}(t, x, y)=-\frac{\sigma}{2} \Delta d^{0} \quad \text { on } \quad \Gamma^{0}
$$

where $\sigma=\int_{\mathbb{R}}\left(\phi_{\xi}^{0}(\xi)\right)^{2} d \xi$. Here we have used (20). 
From the next order term in (18), we know that $\left(v^{1}, p^{0}\right)(t, x, y, \xi)$ satisfy:

$$
\left\{\begin{array}{l}
\eta v_{\xi \xi}^{1}=\left(p_{\xi}^{0}-\mu^{0} \phi_{\xi}^{0}\right) \nabla d^{0} \\
v_{\xi}^{1} \cdot \nabla d^{0}=0
\end{array}\right.
$$

which implies

$$
p_{\xi}^{0}-\mu^{0} \phi_{\xi}^{0}=0 \quad \text { on } \quad \Gamma^{0} .
$$

Integrating in $\xi$ over $(-\infty,+\infty)$ gives

$$
\left[p_{0}\right]=-\sigma \Delta d^{0}, \quad \text { on } \quad \Gamma^{0},
$$

where $[\cdot]$ denotes the jump of a related function on $\Gamma^{0}$.

From the second equation in (26) and the matching condition (14) for $v^{1}$, it follows

$$
\left(\nabla d^{0} \cdot \nabla\right) v_{0}^{ \pm} \cdot \nabla d^{0}=0 .
$$

Now, let us derive the leading order contact angle condition for $\Gamma^{0}=\{x=X(t, y)\}$ at $\{y=0\}$. Take the inner expansion of GNBC in (8), we obtain from the $O\left(\epsilon^{-1}\right)$ order that

$$
\left(\partial_{y} d^{0} \partial_{\xi} \phi^{0}-\frac{\partial \gamma_{w f}}{\partial \phi}\left(\phi^{0}\right)\right) \phi_{\xi}^{0}=0
$$

at $\Gamma^{0} \cap\{y=0\}$. Again, integrating in $\xi$ over $(-\infty,+\infty)$ gives Young's equation:

$$
\cos \alpha_{0}=\frac{\gamma_{-}-\gamma_{+}}{\sigma}
$$

where $\alpha_{0}=\left.\cos ^{-1}\left(-\partial_{y} d^{0}\right)\right|_{y=0, L}$ is the angle between the sharp interface $\Gamma^{0}=$ $\left\{d^{0}(t, x, y)=0\right\}$ and the $x$-axis at $\{y=0, L\}, \sigma=\int_{-\infty}^{+\infty}\left(\partial_{\xi} \phi^{0}\right)^{2} d \xi$ and $\gamma_{ \pm}=\gamma_{w f}( \pm 1)$.

From the leading order inner expansion of the relaxation boundary condition given in (8), we have that at $\Gamma^{0} \cap\{y=0\}$,

$$
\left(\partial_{t} d^{0}+v_{0,1}^{+} \partial_{x} d^{0}\right) \partial_{\xi} \phi^{0}=\partial_{y} d^{0} \partial_{\xi} \phi^{0}-\frac{\partial \gamma_{w f}\left(\phi^{0}\right)}{\partial \phi}=0
$$

which implies

$$
\partial_{t} d^{0}+v_{0,1}^{+} \partial_{x} d^{0}=0, \quad \nabla d^{0} \cdot\left[\nabla \mu_{0}\right]=0, \quad \text { at } \Gamma^{0} \cap\{y=0\} .
$$

2.3. The leading order behavior. We now summarize the results obtained from the inner and outer expansions. The leading profiles of the outer solutions, $\left(\phi_{0}^{ \pm}, \mu_{0}^{ \pm}, v_{0}^{ \pm}, p_{0}^{ \pm}\right)$satisfy the following Hele-Shaw equations coupled with the incompressible Navier-Stokes equations with a free boundary $\Gamma^{0}$ :

$$
\left\{\begin{array}{l}
\phi_{0}^{ \pm}= \pm 1 \\
\Delta \mu_{0}^{ \pm}=0 \\
\partial_{t} v_{0}^{ \pm}+\left(v_{0}^{ \pm} \cdot \nabla\right) v_{0}^{ \pm}+\nabla p_{0}^{ \pm}=\eta \Delta v_{0}^{ \pm} \\
\nabla \cdot v_{0}^{ \pm}=0
\end{array}\right.
$$


The boundary conditions for $\mu_{0}^{ \pm}, v_{0}^{ \pm}$are

$$
\frac{\partial \mu_{0}^{ \pm}}{\partial y}=0, \quad v_{0,2}^{ \pm}=0, \quad \beta v_{0,1}^{ \pm}+\eta \frac{\partial v_{0,1}^{ \pm}}{\partial n}=0,
$$

at the solid boundaries $\{y=0, L\}$, and

$$
\mu_{0}^{ \pm}=-\frac{\sigma}{2} \Delta d^{0}, \quad\left[v_{0}\right]=0, \quad\left(\nabla d^{0} \cdot \nabla\right) v_{0}^{ \pm} \cdot \nabla d^{0}=0, \quad\left[p_{0}\right]=-\sigma \Delta d^{0}
$$

at the interface $\Gamma^{0}$. The dynamics of the interface is given by

$$
\partial_{t} d^{0}+\left(v_{0}^{+} \cdot \nabla\right) d^{0}=\frac{M}{2} \nabla d^{0} \cdot\left[\nabla \mu_{0}\right]
$$

with the contact angle at the boundary being specified by the Young's equation

$$
\cos \alpha_{0}=\frac{\gamma_{-}-\gamma_{+}}{\sigma}
$$

where $\sigma=\int_{\mathbb{R}}\left(\phi_{\xi}^{0}(\xi)\right)^{2} d \xi$ and $\gamma_{ \pm}=\gamma_{w f}( \pm 1)$. Moreover, at the boundary we have

$$
\partial_{t} d^{0}+v_{0,1}^{+} \partial_{x} d^{0}=0
$$

REMARK. (1) The velocity of the interface is not only determined by the local flow velocity $v_{0}$ but also determined by the normal jump of the gradient of the chemical potential which is related to the interfacial mean curvature and surface tension through (34).

(2). Let $\alpha_{1}$ be the leading error between the dynamic angle $\alpha^{\epsilon}$ and the static one $\alpha_{0}$ as given by the Young's equation. From the generalized Navier boundary conditions given in (8), we can deduce that

$$
\alpha_{1}=-\frac{1}{\sigma \sin \alpha_{0}} \int_{-\infty}^{\infty}\left(\frac{\partial^{2} \gamma_{w f}\left(\phi^{0}(\xi)\right)}{\partial \phi^{2}} \phi^{1}-\cos \alpha_{0} \partial_{\xi} \phi^{1}\right) \phi_{\xi}^{0}(\xi) d \xi
$$

at $\Gamma^{0} \cap\{y=0\}$, where $\phi^{1}(t, x, y, \xi)$ is the $O(\epsilon)$-order profile of the inner layer, which is determined by the problem (24). So, the leading angle error $\alpha_{1}$ depends on the structure of the inner layer on the interface. The detail derivation of the formula (37) will be presented in [14].

(3) In [14], we have also studied the problem (6) for the case that the mobility coefficient $M$ depends on the interface thickness $\epsilon$. When $M$ is of the order $O\left(\epsilon^{\gamma}\right)$ for certain $0<\gamma<1$, we show that the leading profiles of the outer solutions satisfy the same free boundary problem for the Hele-Shaw equations coupled with the incompressible Navier-Stokes equations given above. But the interface dynamic equation (35) is replaced by

$$
\partial_{t} d^{0}+\left(v_{0}^{+} \cdot \nabla\right) d^{0}=0, \quad \text { on } \Gamma^{0} .
$$

In this case, the interface $\Gamma^{0}$ moves with the flow speed.

Acknowledgements. We thank T.Z. Qian and P. Sheng for some helpful discussions. This work is partially supported by the Hong Kong RGC Central Allocation Grant CA05/06.SC01 and DAG 04/05.SC17. The work of Ya-Guang Wang is also partially supported by a key project of NSFC under the grant 10531020 and a joint project from the NSAF of China under the grant 10676020. 


\section{REFERENCES}

[1] Alikakos, N. D., Bates, P. W. \& Chen, X., Convergence of the Cahn-Hilliard equation to the Hele-Shaw model, Arch. Rational Mech. Anal., 128 (1994), pp. 165-205.

[2] Briant, A. J. \& Yeomans, J. M., Lattice Boltzmann simulations of contact line motion. II. Binary fluids, Phys. Rev. E, 69 (2004), 031603.

[3] Cahn, J. W. \& Hilliard, J. E., Free energy of a nonuniform system. I. Interfacial free energy, J. Chem. Phys., 28 (1958), p. 258.

[4] CaginalP, G. \& Fife, P., dynamics of layered interfaces arising from phase boundaries, SIAM J. app. Math, 48 (1988), pp. 506-518.

[5] Chen, H. Y., Jasnow, D. \& Vinals, J., Interface and contact line motion in a two phase fluid under shear flow, Phys. Rev. Lett., 85 (2000), p. 1686.

[6] Dussan, V. E. B. \& Davis, S. H., On the motion of a fluid-fluid interface along a solid surface, J. Fluid Mech., 65 (1974), pp. 71-95.

[7] Jacqmin, D., Contact-line dynamics of a diffuse fluid interface, J. Fluid. Mech., 402 (2000), p. 57.

[8] Pego, R. L., Front migration in the nonlinear Cahn-Hilliard equation, Proc. R. Soc. Lond., A, 422 (1989), pp. 261-278.

[9] Qian, T. Z., Wang, X. P. \& Sheng, P., Molecular scale contact line hydrodynamics of immiscible flows, Phys. Rev. E, 68 (2003), 016306.

[10] Qian, T. Z., Wang, X. P. \& Sheng, P., Power-law slip profile of the moving contact line in two-phase immiscible flows, Phys. Rev. Lett., 93 (2004), 094501.

[11] Qian, T. Z., Wang, X. P. \& Sheng, P., Power-law slip profile of the moving contact line in two-phase immiscible flows, Journal of Fluid Mechanics, 564 (2006), pp. 333-360.

[12] Ren, W. \& E, W., Boundary conditions for the moving contact line problem, Phys. Fluids, 19 (2007), 022101(1-15)

[13] Seppecher, P., Moving contact lines in the Cahn-Hilliard theory, Int. J. Engng. Sci., 34 (1996), p. 977.

[14] Wang, X. P. \& Wang, Y. G., Sharp interface limit for a diffusive interface model, preprint 2007. 